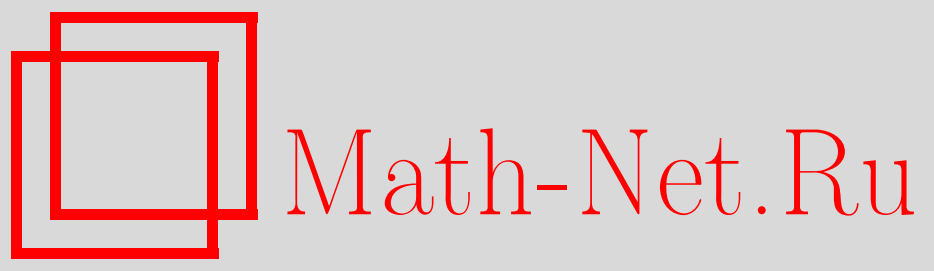

Д. В. Васильев, А. В. Забродин, Самоподобные решения задачи лапласовского роста в полуплоскости, ТMФ, 2011, том 166, номер 1, 28-43

DOI: https://doi.org/10.4213/tmf6593

Использование Общероссийского математического портала Math-Net.Ru подразумевает, что вы прочитали и согласны с пользовательским соглашением http://www . mathnet.ru/rus/agreement

Параметры загрузки:

IP : 54.84 .234 .179

26 апреля 2023 г., 13:39:51

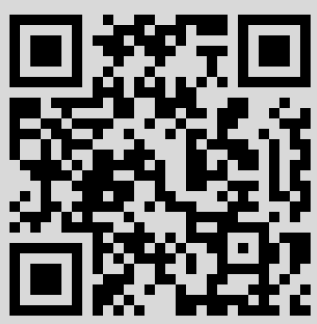




\title{
САМОПОДОБНЫЕ РЕШЕНИЯ ЗАДАЧИ ЛАПЛАСОВСКОГО РОСТА В ПОЛУПЛОСКОСТИ
}

\begin{abstract}
Исследован вариант задачи лапласовского роста в верхней полуплоскости с нулевым поверхностным натяжением. С помощью метода зависящих от времени конформных отображений найдено семейство самоподобных точных решений, которые выражаются в терминах гипергеометрической функции.
\end{abstract}

Ключевые слова: лапласовский рост, интегрируемые системы, конформные отображения, гармонические моменты.

\section{1. ВВЕДЕНИЕ}

В настоящей работе найдены некоторые самоподобные точные решения задачи о лапласовском росте в верхней полуплоскости $\mathbb{H}[1]$. Напомним постановку задачи. Пусть $\gamma$ - гладкая кривая в $\mathbb{H}$ без самопересечений, начинающаяся в точке $x_{-} \in \mathbb{R}$ и заканчивающаяся в точке $x_{+} \in \mathbb{R}$ (предполагается, что $\left.x_{-} \leqslant x_{+}\right)$. Область, ограниченную данной кривой и отрезком $\left[x_{-}, x_{+}\right]$, обозначим через В (в работе [1] такие области были названы “толстыми разрезами"). Пусть кривая $\gamma=\gamma(T)$ эволюционирует с течением времени $T$ согласно закону Дарси:

$$
V_{n}(z)=\frac{1}{2} \partial_{n} \phi(z), \quad z \in \gamma
$$

Здесь $\partial_{n}$ - нормальная производная на границе (нормальный вектор направлен наружу области В), $V_{n}(z)$ - нормальная скорость в точке $z \in \gamma$, а $\phi$ - единственная гармоническая функция в $\mathbb{H} \backslash$ В такая, что $\phi=0$ на $\gamma$ и лучах $\left[-\infty, x_{-}\right],\left[x_{+},+\infty\right]$, лежащих на действительной прямой; $\phi(z)=\operatorname{Im} z+o(1)$ при $\operatorname{Im} z \rightarrow+\infty$.

Как показано в работе [1], процесс роста хорошо определен, если углы $\alpha_{ \pm}$между кривой $\gamma$ и действительной осью в точках $x_{ \pm}$являются острыми. В этом случае углы $\alpha_{ \pm}$и точки $x_{ \pm}$не изменяются с течением времени.

Сравнение такой постановки задачи с обычным лапласовским ростом в верхней полуплоскости показывает, что условия на функцию $\phi$ очень похожи: в стандартной

${ }^{*}$ Институт теоретической и экспериментальной физики, Москва, Россия

${ }^{\dagger}$ Институт биохимической физики им. Н. М. Эммануэля РАН, Москва, Россия. E-mail: zabrodin@itep.ru 
постановке $\phi=0$ на бесконечном контуре, проходящем от левой до правой бесконечности, а при $\operatorname{Im} z \rightarrow+\infty$ функция $\phi \sim \operatorname{Im} z$. Важное различие состоит в том, что только конечная часть линии уровня $\phi=0$, лежащая выше действительной оси, движется по закону Дарси. При этом оставшаяся часть контура, состоящая из лучей действительной оси, остается неподвижной, хотя на этих лучах градиент $\phi$ отличен от нуля.

Часто удобно рассматривать растущую область В как верхнюю половину области $\mathrm{D}=\mathrm{B} \cup \overline{\mathrm{B}}$, симметричной относительно действительной оси, с границей $\Gamma=\gamma \cup \bar{\gamma}$. Здесь $\overline{\mathrm{B}}$ - область в нижней полуплоскости, полученная из В комплексным сопряжением $z \rightarrow \bar{z}$. В дальнейшем мы будем называть такие области симметричными. Таким образом, мы можем расширить задачу (1.1) на всю плоскость “с помощью отражения", т. е. положив

$$
V_{n}(z)=\left\{\begin{aligned}
\frac{1}{2} \partial_{n} \phi(z), & z \in \gamma \\
-\frac{1}{2} \partial_{n} \phi(z), & z \in \bar{\gamma}
\end{aligned}\right.
$$

где $\phi$ - такая гармоническая функция в $\mathbb{C} \backslash \mathrm{D}$, что $\phi(\bar{z})=-\phi(z) ; \phi=0$ на $\Gamma ; \phi(z)=$ $\operatorname{Im} z+o(1)$ при $\operatorname{Im} z \rightarrow \pm \infty$.

\section{2. ФОРМУЛИРОВКА В ТЕРМИНАХ КОНФОРМНЫХ ОТОБРАЖЕНИЙ}

Мы применим обычный для задач с подвижными границами прием и переформулируем нашу задачу в терминах зависящего от времени конформного отображения на некоторую стандартную область из области $\mathbb{C} \backslash \mathrm{D}(T)$, где нужно решить уравнение Лапласа (или в терминах обратного отображения). В нашем случае есть два выделенных выбора стандартной области - верхняя полуплоскость и внешность единичного круга.

2.1. Верхняя полуплоскость. Этот выбор является естественным, если мы рассматриваем первоначально поставленную задачу в верхней полуплоскости, не распространяя ее на нижнюю полуплоскость с помощью отражения. Пусть $p(z)-$ конформное отображение из $\mathbb{H} \backslash$ В (в “физической" $z$-плоскости) в $\mathbb{H}$ (в "математической" $p$-плоскости). Это отображение схематически показано на рис. 1. Мы нормируем отображение условием на ряд Лорана функции $p(z)$ на бесконечности (“гидродинамическая” нормировка):

$$
p(z)=z+\frac{u_{1}}{z}+\sum_{k=2}^{\infty} u_{k} z^{-k}, \quad|z| \rightarrow \infty .
$$

С учетом данной нормировки отображение единственно. Верхняя часть границы $($ кривая $\gamma)$ отображается в интервал $\left[p_{-}, p_{+}\right]$на действительной оси, а лучи на действительной оси, лежащие вне $\mathrm{B}$, отображаются на лучи $\left[-\infty, p_{-}\right]$и $\left[p_{+}, \infty\right]$. Следовательно, все коэффициенты $u_{k}$ - действительные числа. Первый коэффициент $u_{1}$ называется емкостью области В. Он всегда положителен. Нам также потребуется 


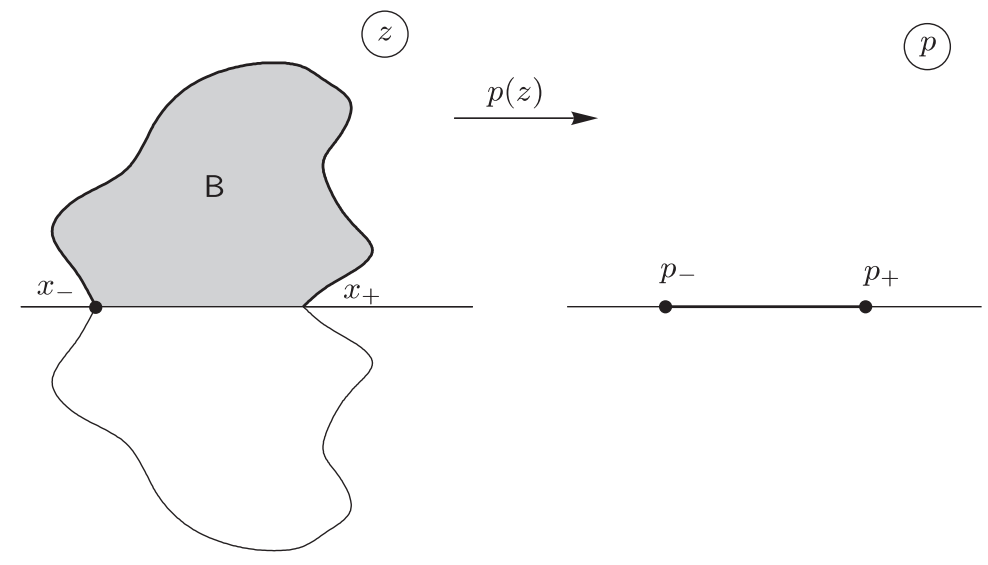

Рис. 1. Конформное отображение $p(z)$. Также показан зеркальный образ области В в нижней полуплоскости.

обратное отображение $z(p)$. Оно может быть представлено в виде обратного ряда Лорана

$$
z(p)=p-\frac{u_{1}}{p}+\sum_{k=2}^{\infty} a_{k} p^{-k}, \quad|p| \rightarrow \infty,
$$

с действительными коэффициентами $a_{k}$, связанными с $u_{k}$ полиномиальными соотношениями. Ряд сходится при достаточно больших $|p|$.

Из сказанного ясно, что гармоническая функция $\operatorname{Im} p(z)$ удовлетворяет всем требованиям, налагаемым на $\phi(z)$, следовательно, $\phi(z)=\operatorname{Im} p(z)$. Легко видеть, что $\partial_{n} \operatorname{Im} p(z)=\left|p^{\prime}(z)\right|$ на $\gamma$, следовательно, закон Дарси может быть записан в виде $V_{n}(z)=\left|p^{\prime}(z)\right| / 2$.

Принцип симметрии Шварца позволяет распространить эту формулировку задачи на всю плоскость следующим образом: функция $z(p)$ может быть аналитически продолжена на нижнюю полуплоскость как $\overline{z(\bar{p})}$. Аналитически продолженная функция конформно отображает комплексную плоскость с разрезом по действительной оси от $p_{-}$до $p_{+}$на внешность симметричной области $\mathrm{D}=\mathrm{B} \cup \overline{\mathrm{B}}$. Соответственно, обратная функция $p(z)$ удовлетворяет соотношениям $p(\bar{z})=\overline{p(z)}$ и $\partial_{n} \operatorname{Im} p(z)=-\left|p^{\prime}(z)\right|$ на $\bar{\gamma}$. Эволюция всего замкнутого контура $\Gamma=\gamma \cup \bar{\gamma}$ может быть единообразно описана законом $V_{n}(z)=\left|p^{\prime}(z)\right| / 2$ для любого $z \in \Gamma$.

Отображение $z(p)$ играет ключевую роль во вложении задачи в бездисперсионную иерархию Кадомцева-Петвиашвили, которое было найдено в работе [1]. При этом данная параметризация оказывается довольно неудобной для нахождения явных решений. Это связано с тем, что симметричным образом достроенная область в математической плоскости сингулярна (плоскость с разрезом) и, более того, зависит от времени. Существует другой выбор стандартной области, менее естественный с точки зрения интегрируемых систем, но более подходящий для нахождения явных решений.

2.2. Внешность единичного круга. В данном случае удобно с самого начала работать с симметричным образом достроенной областью. Пусть $f(w)$ - конформное 
отображение из внешности единичного круга во внешность симметричной области $\mathrm{D}$ такое, что $f(\infty)=\infty$ и $f^{\prime}(\infty)=r>0$; таким образом, ряд Лорана в бесконечности имеет вид $f(w)=r w+u_{0}+O(1 / w)$, и все его коэффициенты действительны. Коэффициент $r$ называется (внешним) конформным радиусом области D. Связь с ранее рассмотренным конформным отображением такова: пусть $w(z)$ - обратная функция к $f(w)$, тогда

$$
p(z)=r\left(w(z)+\frac{1}{w(z)}\right)+u_{0}, \quad r=\frac{p_{+}-p_{-}}{4}, \quad u_{0}=\frac{p_{+}+p_{-}}{2} .
$$

Перепишем закон Дарси в виде динамического уравнения относительно функции $f(w)=f(w, T)$. Для этого нам потребуется кинематическое соотношение, которое можно вывести непосредственно. Пусть $z(\sigma, T)=x(\sigma, T)+i y(\sigma, T)-$ произвольная параметризация контура, здесь $\sigma$ - монотонно возрастающая функция длины дуги. Тогда

$$
V_{n}=\frac{d \sigma}{d l}\left(\partial_{T} x \cdot \partial_{\sigma} y-\partial_{T} y \cdot \partial_{\sigma} x\right)=\frac{1}{2 i} \frac{d \sigma}{d l}\left(\partial_{T} \bar{z} \cdot \partial_{\sigma} z-\partial_{T} z \cdot \partial_{\sigma} \bar{z}\right),
$$

где $d l=|d z|=\sqrt{(d x)^{2}+(d y)^{2}}$ - линейный элемент вдоль контура. Примем соглашение, что скорость $V_{n}(z)$ положительна, когда контур в окрестности точки $z$ движется вправо относительно направления возрастания $\sigma$. Положим $\sigma=-i \ln w$, $z=f(w, T), \bar{z}=f(1 / w, T)$, тогда из кинематической формулы получим

$$
V_{n}(f(w))=\frac{1}{2 i} \frac{d w}{|d f(w)|}\left(\partial_{T} f(1 / w) \cdot \partial_{w} f(w)-\partial_{T} f(w) \cdot \partial_{w} f(1 / w)\right), \quad|w|=1 .
$$

С другой стороны, из закона Дарси следует, что

$$
V_{n}(f(w))=\frac{1}{2} \frac{|d p / d w|}{|d f / d w|}, \quad|w|=1 .
$$

Приравнивая правые части двух равенств для $V_{n}(f(w))$ и используя соотношение $d p / d w=r\left(1-1 / w^{2}\right)$, получим уравнение

$$
w \partial_{w} f(w) \cdot \partial_{T} f(1 / w)-w \partial_{w} f(1 / w) \cdot \partial_{T} f(w)=r|w-1 / w|, \quad|w|=1 .
$$

Данное уравнение является основным динамическим уравнением задачи, сформулированной в терминах конформных отображений.

Полезно сравнить полученное уравнение с уравнением лапласовского роста во всей плоскости (т. е. без условия, что $\phi$ обращается в нуль на действительной оси) с тем же самым источником на бесконечности. В этом случае динамика с необходимостью разрушит зеркальную симметрию, следовательно, функцию $f(1 / w)$ необходимо заменить на $\bar{f}(1 / w) \equiv \overline{f(1 / \bar{w})}$. При этом правая часть уравнения также должна быть заменена на $-i r(w-1 / w)$.

Для нахождения точных решений важен случай, когда на некотором интервале времен $T$ функцию $f(w, T)$ можно аналитически продолжить через единичную окружность так, чтобы она была аналитична не только во внешности единичного круга, но и в несколько большей области, захватывающей его границу. Заметим, однако, что такое аналитическое продолжение невозможно через точки $w= \pm 1$, 
являющиеся прообразами двух угловых точек, следовательно, в них $f(w)$ не аналитична. Предполагая, что она аналитична в остальных точках единичного круга, мы можем аналитически продолжить уравнение (2.4) следующим образом:

$$
w \partial_{w} f(w) \cdot \partial_{T} f(1 / w)-w \partial_{w} f(1 / w) \cdot \partial_{T} f(w)=\left\{\begin{aligned}
-i r(w-1 / w), & \operatorname{Im} w>0 \\
i r(w-1 / w), & \operatorname{Im} w<0
\end{aligned}\right.
$$

Заметим, что аналитические продолжения в верхнюю и нижнюю полуплоскости различаются.

\section{3. ФОРМУЛИРОВКА В ТЕРМИНАХ ФУНКЦИИ ШВАРЦА}

Известно [2], что стандартная задача лапласовского роста во всей плоскости может быть проинтегрирована в терминах функции Шварца. Для аналитических контуров функция Шварца $S(z)$ определена как аналитическое продолжение функции $\bar{z}$ с контура. Другими словами, функция Шварца кривой Г - это такая аналитическая функция $S(z)$, что $\bar{z}=S(z)$ для $z \in \Gamma$ (подробности см. в [3]). В случае кривой, зависящей от времени, функция Шварца также зависит от времени: $S=S(z, T)$.

В нашей задаче контур $\gamma \cup \bar{\gamma}$ не аналитичен, так как он обязательно содержит два излома в точках $x_{-}, x_{+}$. Тем не менее, если контур $\gamma$ аналитичен, то его функция Шварца (также обозначаемая через $S(z))$ хорошо определена в полосе, толщина которой стремится к нулю в окрестности концов кривой $x_{ \pm}$. Таким образом, $\bar{z}=S(z)$ для $z \in \gamma$ или, что тоже самое, $\bar{z}=\overline{S(\bar{z})}$ для $z \in \bar{\gamma}$. Последняя формула означает, что $\bar{S}(z)=\overline{S(\bar{z})}$ - функция Шварца для комплексно-сопряженной кривой $\bar{\gamma}$. Таким образом, можно ожидать, что наша задача интегрируется с помощью двух (равноправных) функций Шварца $S(z)$ и $\bar{S}(z)$. Ниже, исключительно для краткости, будем называть пару $(S(z), \bar{S}(z))$ функцией Шварца кусочно-аналитического контура $\Gamma=\gamma \cup \bar{\gamma}$. В математической $w$-плоскости функция Шварца позволяет связать функции $f(w)$ и $f(1 / w)$ в областях, где они обе аналитичны:

$$
f(w)= \begin{cases}\bar{S}(f(1 / w)), & \operatorname{Im} w>0 \\ S(f(1 / w)), & \operatorname{Im} w<0\end{cases}
$$

Заметим, что второе равенство (при $\operatorname{Im} w<0$ ) получается из первого с помощью комплексного сопряжения.

В полной аналогии с задачей лапласовского роста на всей плоскости процесс (1.1) в верхней полуплоскости определяется уравнением $i \partial_{T} S=\partial_{z} p$. Расширяя его на всю плоскость с помощью отражения (комплексного сопряжения), для всех $z \in \mathbb{C} \backslash \mathrm{D}(T)$ получим

$$
\begin{aligned}
& i \partial_{T} S(z, T)=\partial_{z} p(z, T), \quad \operatorname{Im} z>0, \\
& i \partial_{T} \bar{S}(z, T)=-\partial_{z} p(z, T), \quad \operatorname{Im} z<0 .
\end{aligned}
$$

Данные соотношения удобно переписать в терминах кусочно-аналитической функции

$$
M(z)=\left\{\begin{aligned}
i(S(z)-z), & \operatorname{Im} z>0 \\
-i(\bar{S}(z)-z), & \operatorname{Im} z<0
\end{aligned}\right.
$$


введенной в статье [1]. По построению эта функция является аналитическим продолжением функции $2|\operatorname{Im} z|$ с контура Г. Эквивалентным образом запишем $S(z)=$ $z-i M(z)$ для $\operatorname{Im} z>0$ и $\bar{S}(z)=z+i M(z)$ для $\operatorname{Im} z<0$ или, на математической $w$-плоскости,

$$
f(w)= \begin{cases}f(1 / w)+i M(f(1 / w)), & \operatorname{Im} w>0 \\ f(1 / w)-i M(f(1 / w)), & \operatorname{Im} w<0 .\end{cases}
$$

В терминах функции $M=M(z, T)$ динамическое уравнение (3.2) принимает вид

$$
\partial_{T} M(z, T)=\partial_{z} p(z, T)
$$

Заметим, что функция $\partial_{z} p(z, T)$ голоморфна в области $\mathbb{C} \backslash \mathrm{D}(T)$ и имеет разложение $\partial_{z} p(z, T)=1+O\left(1 / z^{2}\right)$ в бесконечности.

Функция $M(z)$ единственным образом разлагается как $M(z)=M_{+}(z)-M_{-}(z)$, где функция $M_{+}$аналитична в $\mathrm{D}$, а $M_{-}$аналитична в $\mathbb{C} \backslash \mathrm{D}$ и равна нулю в бесконечности. Это разложение задается интегралом типа Коши:

$$
M_{+}(z)=\frac{1}{2 \pi i} \oint_{\partial \mathrm{D}} \frac{M\left(z^{\prime}\right)}{z^{\prime}-z} d z^{\prime}=\frac{1}{\pi i} \oint_{\partial \mathrm{D}} \frac{\left|\operatorname{Im} z^{\prime}\right|}{z^{\prime}-z} d z^{\prime}, \quad z \in \mathrm{D}
$$

(и таким же интегралом для $M_{-}(z)$ с $z$, лежащим вне D). Функция $M_{+}$аналитична всюду в D и согласно нашему предположению может быть аналитически продолжена через дуги $\gamma$ и $\bar{\gamma}$ везде, за исключением их концов на действительной оси, в которых она имеет особенности. Покажем, что $M_{+}(z)$ является производящей функцией для интегралов движения нашей задачи. Действительно, вычислив ее производную по времени, получим

$$
\partial_{T} M_{+}(z)=\frac{1}{\pi i} \oint_{\partial \mathrm{D}} \frac{\operatorname{sign}\left(\operatorname{Im} z^{\prime}\right) V_{n}\left(z^{\prime}\right)}{z-z^{\prime}}\left|d z^{\prime}\right|
$$

(подробнее см. в [1]). Подставляя $V_{n}(z)=\left|p^{\prime}(z)\right| / 2$ и замечая, что $d p(z)$ чисто действительно на Г, получим

$$
\partial_{T} M_{+}(z)=\frac{1}{2 \pi i} \oint_{\partial \mathrm{D}} \frac{d p\left(z^{\prime}\right)}{z^{\prime}-z}=1
$$

Здесь ненулевой вклад дает только вычет в бесконечности, так как $p^{\prime}(z)$ голоморфна вне D, а $z$ находится внутри D. Таким образом, приходим к важному уравнению

$$
\partial_{T} M_{+}(z, T)=1
$$

позволяющему получить бесконечный набор интегралов движения, разлагая $M_{+}(z)$ в ряд по $z$.

В случае общего положения, когда $x_{-} \neq x_{+}$, можно разложить $M_{+}(z)$ в ряд Тейлора в точке на действительной оси, лежащей внутри D на интервале $\left(x_{-}, x_{+}\right)$. Выбрав координаты так, что $x_{-}<0<x_{+}$, можно разложить ее в начале координат:

$$
M_{+}(z)=T+\sum_{k=2}^{\infty} k T_{k} z^{k-1}
$$

2 Теоретическая и математическая физика, т. 166, № 1, 2011 г. 
Из уравнения (3.8) видно, что коэффициенты $T_{k}, k \geqslant 2$, сохраняются: $\partial_{T} T_{k}=0$. Они являются гармоническими моментами области $\mathbb{H} \backslash \mathrm{B}$, определенными следующим образом:

$$
T_{k}=\frac{1}{\pi i k} \oint_{\mathrm{D}}|\operatorname{Im} z| z^{-k} d z=\frac{2}{\pi k} \int_{\mathbb{H} \backslash \mathrm{B}} \operatorname{Im}\left(z^{-k}\right) d^{2} z, \quad k \geqslant 2 .
$$

Можно также рассмотреть вырожденные конфигурации с $x_{-}=x_{+}=0$. В этом случае функция $M_{+}(z)-T$ также сохраняется, но ее нельзя представить в виде ряда Тейлора в нуле, вследствие чего моменты (3.10) плохо определены.

\section{4. САМОПОДОБНЫЕ РЕШЕНИЯ}

Самоподобные решения характеризуются свойством

$$
f(w, T)=r(T) f(w),
$$

которое означает, что эволюция по времени является растяжением в плоскости $z$. Вследствие того что эволюция оставляет неподвижными точки $x_{ \pm}$, самоподобные решения существуют только тогда, когда $x_{-}=x_{+}=0$. Это является вырожденным случаем, упоминавшимся ранее. Точные самоподобные решения задачи лапласовского роста в радиальной постановке и внутри сектора были изучены в работах [4]-[9].

\section{1. Общие соотношения.}

Дифференциальное уравнение для $f(w)$. Подставляя самоподобный анзац (4.1) в (2.5), видим, что переменные разделяются: $r(T)=T / A$ и

$$
w \partial_{w} f(w) \cdot f(1 / w)-w \partial_{w} f(1 / w) \cdot f(w)=A\left\{\begin{aligned}
-i(w-1 / w), & \operatorname{Im} w>0 \\
i(w-1 / w), & \operatorname{Im} w<0
\end{aligned}\right.
$$

где $A$ - постоянная. Последнее равенство может рассматриваться как соотношение для вронскиана дифференциального уравнения второго порядка вида

$$
A_{2}(w) \psi^{\prime \prime}+A_{1}(w) \psi^{\prime}+A_{0}(w) \psi=0 .
$$

Если $\psi_{1}, \psi_{2}$ - два линейно независимых решения, то их вронскиан $W=\psi_{1} \psi_{2}^{\prime}-\psi_{2} \psi_{1}^{\prime}$ удовлетворяет соотношению

$$
\partial_{w} \ln W(w)=-\frac{A_{1}(w)}{A_{2}(w)}
$$

В нашем случае $W= \pm i A\left(1-1 / w^{2}\right)$ и

$$
\partial_{w} \ln W=\frac{1}{w+1}+\frac{1}{w-1}-\frac{2}{w}
$$

(заметим, что это выражение одинаково для обеих полуплоскостей), а функции $f(w)$ и $f(1 / w)$ - два решения обыкновенного дифференциального уравнения

$$
f^{\prime \prime}-\left(\frac{1}{w+1}+\frac{1}{w-1}-\frac{2}{w}\right) f^{\prime}+\widetilde{V}(w) f=0
$$




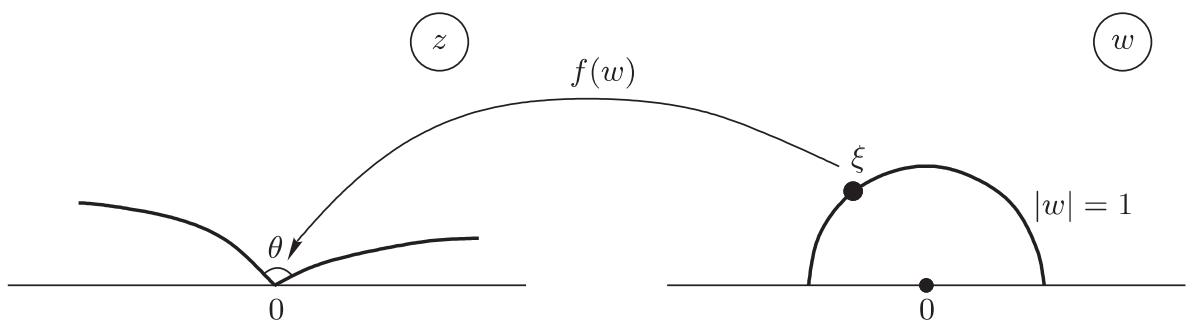

Рис. 2. Угловая точка в начале координат.

с пока неопределенной функцией $\widetilde{V}(w)$ или уравнения

$$
w^{2} \partial_{w}^{2} f-\frac{2 w}{w^{2}-1} \partial_{w} f+V(w) f=0
$$

(здесь $\left.V(w)=w^{2} \widetilde{V}(w)\right)$.

Требование, что $f(1 / w)$ является вторым решением уравнения (4.3), приводит к соотношению $V(1 / w)=V(w)$, а из поведения на бесконечности $f(w)=w+O(1)$ следует, что $V(w)=O\left(1 / w^{2}\right)$. Более точно, если $f(w)=w+a_{0}+a_{1} 1 / w+O\left(1 / w^{2}\right)$, то с помощью простого вычисления можно получить, что $V(w)=2\left(1-a_{1}\right) / w^{2}+O\left(1 / w^{3}\right)$. Далее, $f(w)$ регулярна вне единичного круга (за исключением простого полюса на бесконечности) и $V(1 / w)=V(w)$, следовательно, $V(w)$ может иметь сингулярности только на единичной окружности. Доказательство аналогично случаю самоподобного лапласовского роста в клине [6]. Предположим, например, что $V$ имеет полюс в некоторой точке $\xi$ вне единичного круга. Тогда этот полюс можно было бы скомпенсировать, только наложив условие $f(\xi)=0$, что в свою очередь означало бы, что $\xi$ на самом деле лежит на единичной окружности. Действительно, если 0 образ такой точки $w$, что $|w| \geqslant 1$, то эта точка является сингулярной граничной точкой, следовательно, ее прообраз лежит на единичной окружности.

Здесь мы не будем изучать вопрос о наиболее общем виде сингулярностей на единичном круге, разрешенных для функции $V(w)$, и ограничимся случаем рациональной функции $V(w)$. Предположим, что граница испытывает излом с углом $\theta$, когда точка $w$, двигающаяся по единичной окружности, проходит через точку $\xi \neq \pm 1$ (см. рис. 2), тогда в окрестности $\xi$ конформное отображение имеет вид $f(w) \propto(w-\xi)^{\theta / \pi}$ (при этом мы предполагаем, что граница может быть не гладкой только в начале координат, т. е. $f(\xi)=0)$. Подставляя асимптотику в уравнение (4.3), получим

$$
V(w)=\frac{(\theta / \pi)(1-\theta / \pi) \xi^{2}}{(w-\xi)^{2}}(1+O(w-\xi)), \quad w \rightarrow \xi
$$

Заметим, что главная часть одинакова для углов $\theta$ и $\pi-\theta$. Ясно, что точно такой же полюс с тем же $\theta$ находится в комплексно-сопряженной точке $\bar{\xi}$. Полюсы в точках $\xi= \pm 1$ должны рассматриваться отдельно. В этом случае

$$
V(w)=\frac{4(\theta / \pi)(1-\theta / \pi)}{(w \mp 1)^{2}}(1+O(w \mp 1)), \quad w \rightarrow \pm 1,
$$


где через $\theta$ теперь обозначен угол между действительной осью и ближайшим куском границы, выходящим из начала координат.

Подытожим свойства функции $V(w)$ :

а) $V(w)$ - рациональная функция переменной $w$ такая, что $V(w)=V(1 / w)$;

б) единственными особенностями $V(w)$ являются полюсы второго порядка на единичной окружности с главной частью, задаваемой соотношениями $(4.4),(4.5)$;

в) $V(w)=O\left(1 / w^{2}\right)$ при $w \rightarrow \infty$.

В ряде случаев эти условия позволяют однозначно зафиксировать вид $V(w)$ (см. примеры ниже). Для нахождения конформного отображения $f(w)$ необходимо решить уравнение (4.3), выбрать решение, удовлетворяющее условию $f(w)=w+O(1)$ при $w \rightarrow \infty$, и проверить, что отображение на самом деле конформно, т. е. что $f^{\prime}(w) \neq 0$ для всех $w$ вне единичного круга. В действительности достаточно проверить, что если $\varphi$ меняется от 0 до $2 \pi$, то $f\left(e^{i \varphi}\right)$ обходит границу $\mathrm{D}$ ровно один раз против часовой стрелки (принцип аргумента, см., например, книгу [10]). В самом деле, число нулей аналитической функции $f^{\prime}(w)$ вне единичного круга дается интегралом $-(1 / 2 \pi) \oint_{|w|=1} d \arg f^{\prime}(w)$ (более точно, чтобы регуляризовать возможные особенности в прообразах угловых точек, необходимо рассматривать интеграл $-(1 / 2 \pi) \oint_{|w|=e^{\epsilon}} d \arg f^{\prime}(w)$ при $\epsilon \rightarrow+0$.) C другой стороны, так как $f$ осуществляет конформное отображение в окрестности единичного круга, нормальный вектор к границе при отображении поворачивается на угол $\arg f^{\prime}(w)$, т. е. $\arg f^{\prime}(w)=$ $\phi(f(w))-\arg w$, где $\phi$ - угол между нормальным вектором к границе, направленным наружу области $\mathrm{D}$, и действительной осью. Из этого ясно, что $\arg f^{\prime}(w)$ не меняется, когда $w$ делает полный оборот вдоль единичной окружности, и, следовательно, $f^{\prime}(w)$ не может обратиться в нуль вне единичной окружности.

Вид функции $M_{+}(z)$. Для конформного отображения $p(z, T)$ самоподобие означает, что $p(z, T)=r(T) p(z / r(T))$, и аналогичное равенство справедливо для $M$-функции: $M(z, T)=r(T) M(z / r(T))$. Подставляя это выражение в (3.5), мы получим, что $\dot{r}=1 / A$, т. е. $r=T / A$, и

$$
M(z)-z M^{\prime}(z)=A p^{\prime}(z) .
$$

Функция $M(z)$ здесь является $M$-функцией области с конформным радиусом $r=1$, т. е. при $T=A$. Проинтегрировав обе части равенства по границе с ядром Коши, получим следующее условие на $M_{+}$:

$$
M_{+}(z)-z M_{+}^{\prime}(z)=A
$$

с общим решением $M_{+}(z)=c z+A$, или

$$
M_{+}(z, T)=c z+T
$$

где $c$ - произвольное действительное число. Важно отметить, что в рассматриваемом случае область D состоит по крайней мере из двух несвязных кусков с границами, пересекающимися в начале координат; таким образом, постоянная $c$ может быть разной в разных кусках области D.

\section{2. Примеры.}

Однолепестковое решение. Простейшим примером является однолепестковая конфигурация, растущая в верхней полуплоскости, симметричная относительно мнимой оси (рис. 3). Пусть $\alpha$ - угол между касательной в начале координат и действи- 


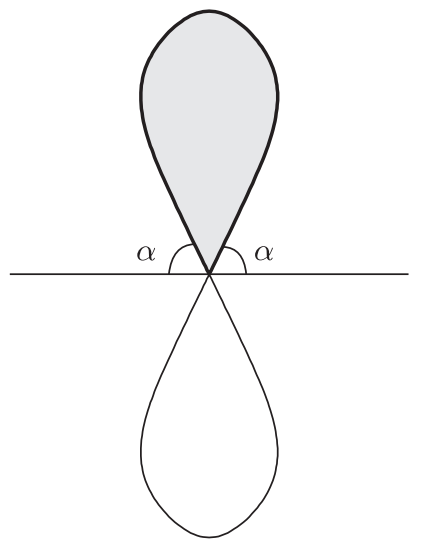

a

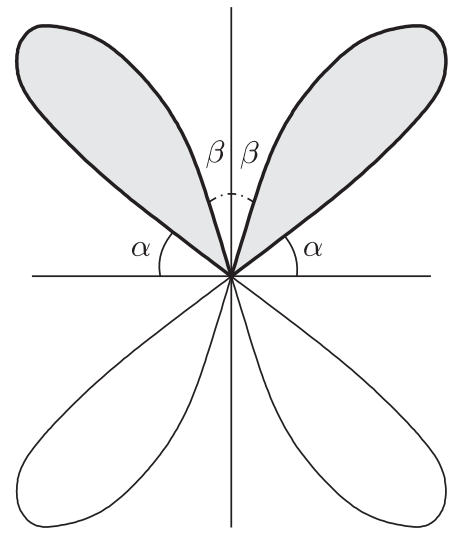

б

Рис. 3. Однолепестковое решение (а) и двухлепесковое решение (б). Также показаны отражения лепестков в нижней полуплоскости.

тельной осью, тогда приведенные в предыдущем пункте условия на функцию $V(w)$ однозначно фиксируют ее:

$$
V(w)=\frac{16 \alpha}{\pi}\left(1-\frac{\alpha}{\pi}\right) \frac{w^{2}}{\left(w^{2}-1\right)^{2}} .
$$

После подстановки $w=e^{x}, f(w)=\operatorname{sh}^{1 / 2} x \cdot \psi(x)$ уравнение (4.3) принимает вид

$$
\psi^{\prime \prime}+\frac{\gamma(1-\gamma)}{\operatorname{sh}^{2} x} \psi=\frac{1}{4} \psi
$$

где

$$
\gamma=\frac{2 \alpha}{\pi}-\frac{1}{2}, \quad-\frac{1}{2}<\gamma<\frac{1}{2}
$$

Его решение с требуемыми условиями на бесконечности дается формулой

$$
\psi(x)=e^{x / 2}\left(1-e^{-2 x}\right)_{2}^{\gamma} F_{1}\left(\begin{array}{c}
\gamma, \gamma-1 / 2 \\
1 / 2
\end{array} ; e^{-2 x}\right) .
$$

Гипергеометрическая функция с такими параметрами может быть выражена через элементарные функции:

$$
{ }_{2} F_{1}\left(\begin{array}{c}
\gamma, \gamma-1 / 2 \\
1 / 2
\end{array} z^{2}\right)=\frac{1}{2}\left[(1+z)^{-2 \gamma}+(1-z)^{-2 \gamma}\right] .
$$

Для $f(w)$ мы, следовательно, получим

$$
f(w)=\frac{1}{2} \sqrt{w^{2}-1}\left((1-1 / w)^{\gamma}(1+1 / w)^{1-\gamma}+(1+1 / w)^{\gamma}(1-1 / w)^{1-\gamma}\right) .
$$

При $\gamma=0(\alpha=\pi / 4)$ данное решение переходит в $f(w)=\sqrt{w^{2}-1}$ из работы [1]. Граница такой области является лемнискатой Бернулли, задаваемой уравнением 


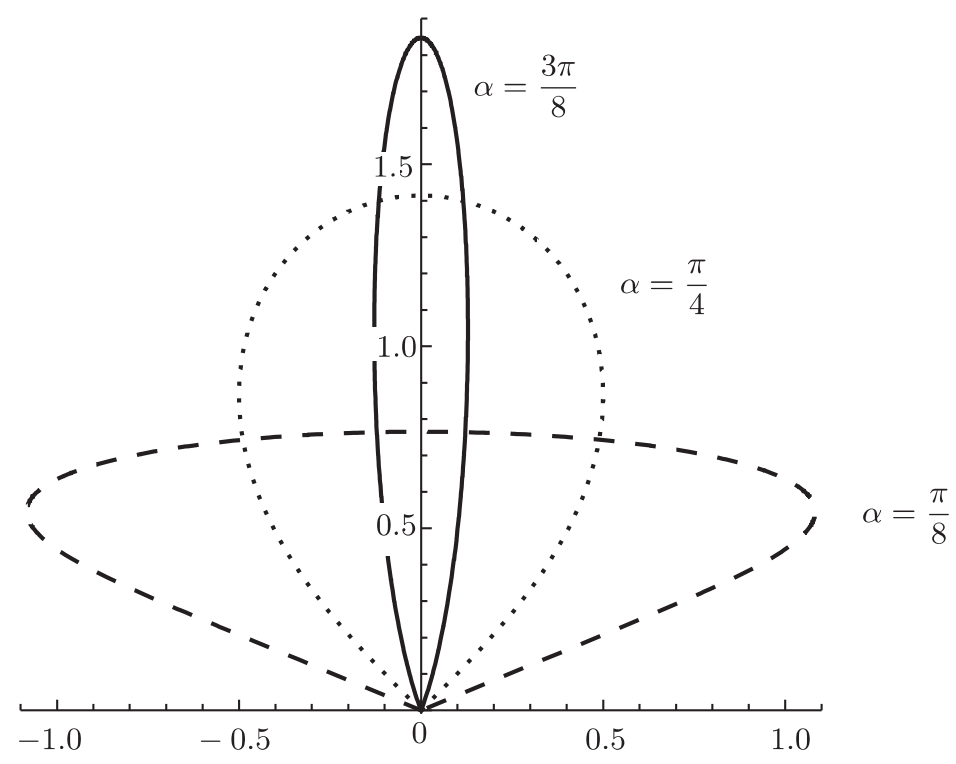

Рис. 4. Однолепестковые решения при разных $\alpha$.

$\left(x^{2}+y^{2}\right)^{2}=2\left(y^{2}-x^{2}\right)$ в декартовых координатах или уравнением $r^{2}=-2 \cos 2 \theta$ в полярных (см. рис. 4, пунктирная линия). Используя принцип аргумента, можно показать, что $f^{\prime}(w) \neq 0$ при $|w| \geqslant 1$ для всех $\gamma$, лежащих между $-1 / 2$ и $1 / 2$, т. е. мы получили непрерывное семейство однолепестковых самоподобных решений с углами $0 \leqslant \alpha<\pi / 2$. Примеры для $\alpha=\pi / 8$ и $\alpha=3 \pi / 8$ также показаны на рис. 4.

Вывод однолепесткового решения с помощью интегрального уравнения. Приведенный выше результат также может быть получен другим способом: с использованием вытекающего из свойств $M$-функции интегрального уравнения для конформного отображения $f(w)$. Функция $M_{+}$однолепесткового решения имеет только один разрез, совпадающий со всей действительной осью. Вследствие соотношения (4.6) скачок функции $M_{+}$на разрезе должен быть линейной функцией. Коэффициент можно определить путем анализа локального поведения в начале координат. Действительно, пусть $e^{i \theta(z)}=d z /|d z|$ - единичный тангенциальный вектор к кривой в точке $z$, представленный как комплексное число. Тогда по определению функции Шварца $S^{\prime}(z)=e^{-2 i \theta(z)}$ для $z$, лежащих на кривой в верхней полуплоскости, и $\bar{S}^{\prime}(z)=e^{-2 i \theta(z)}$ для $z$, лежащих на кривой в нижней полуплоскости. Эти формулы позволяют вычислить скачок функции $M^{\prime}$, равный скачку функции $M_{+}^{\prime}$ на действительной оси. Используя (3.3), найдем

$$
\left[M^{\prime}\right]:=M_{\mathrm{up}}^{\prime}-M_{\text {down }}^{\prime}=\frac{\left(2-S_{\mathrm{up}}^{\prime}-\bar{S}_{\mathrm{down}}^{\prime}\right)}{i}=\frac{2-e^{-2 i \alpha}-e^{2 i \alpha}}{i}=-4 i \sin ^{2} \alpha .
$$

Таким образом, в верхней полуплоскости $M_{+}=-2 i z \sin ^{2} \alpha+A$, а в нижней полуплоскости $M_{+}=2 i z \sin ^{2} \alpha+A$.

Используя формулу (3.1), мы можем выразить сумму значений функции $f(w)$ на противоположных берегах разреза, проходящего от -1 до 1 в $w$-плоскости. Положим 


$$
\begin{aligned}
& \{f(w)\}=f(w+i 0)+f(w-i 0) \text {, тогда } \\
& \quad\{f(w)\}=2 f(1 / w)+i\left[M_{+}(f(1 / w))\right]=\left(2-4 \sin ^{2} \alpha\right) f(1 / w)=2 \cos 2 \alpha \cdot f(1 / w),
\end{aligned}
$$

где использовано соотношение

$$
\left[M_{+}(f(w))\right]=M_{+}(f(w+i 0))-M_{+}(f(w-i 0))=-4 i \sin ^{2} \alpha \cdot f(w) .
$$

Мы получили уравнение

$$
\{f(w)\}=2 \cos 2 \alpha \cdot f(1 / w), \quad w \in[-1,1]
$$

определяющее конформное отображение. Данное уравнение может быть преобразовано в интегральное. Для этого воспользуемся подстановкой

$$
g(w)=\frac{f(w)}{\sqrt{w^{2}-1}},
$$

позволяющей перейти от среднего значения функции $f$ на разрезе к скачку функции $g$. Мы выбираем ветвь квадратного корня так, чтобы функция $\sqrt{w^{2}-1}=$ $w \sqrt{1-1 / w^{2}}$ была нечетной функцией вне единичного круга. Из симметрии лепестка вытекает условие $f(-w)=-f(w)$; таким образом, $g(-w)=g(w)$. В терминах функции $g$ уравнение (4.12) принимает вид

$$
[g(w)]=-2 i \cos 2 \alpha \frac{g(1 / w)}{w}, \quad w \in[-1,1] .
$$

Функция $g$ аналитична всюду в $w$-плоскости за исключением двух точек ветвления $w= \pm 1$. При этом скачок на разрезе $[-1,1]$ задается формулой (4.13). Принимая во внимание, что $g(\infty)=1$, мы можем представить эту функцию в виде интеграла типа Коши вдоль разреза:

$$
g(w)=1-\frac{2 i \cos 2 \alpha}{2 \pi i} \int_{-1}^{1} \frac{g(1 / x)}{x(x-w)} d x .
$$

В результате, используя тот факт, что $g$ - четная функция, мы приходим к интегральному уравнению

$$
g(w)=1-\frac{2 \cos 2 \alpha}{\pi} \int_{0}^{1} \frac{g(1 / x)}{x^{2}-w^{2}} d x .
$$

Мы знаем, что $f(w) \propto(w-1)^{2 \alpha / \pi}$ в окрестности $w=1$, и, следовательно, ищем решение, ведущее себя, как $g(w) \propto(w-1)^{(2 \alpha / \pi)-1 / 2}$, в окрестности $w=1$ и аналогично в окрестности $w=-1$. Заметим, что показатель степени совпадает с $\gamma$ в (4.9).

Сначала рассмотрим случай положительной $\gamma$. После подстановки $g(w)=(1-$ $\left.1 / w^{2}\right)^{\gamma} G\left(1 / w^{2}\right)$, используя то, что $g( \pm 1)=0$ (при положительных $\gamma$ ), приходим к уравнению

$$
(1-x)^{\gamma-1} G(x)=\frac{\sin \pi \gamma}{\pi} \int_{0}^{1} \frac{t^{-1 / 2}(1-t)^{\gamma-1} G(t)}{1-x t} d t
$$


Сравнив его с уравнением (42) из работы [9], можно немедленно выписать решение

$$
G(t)={ }_{2} F_{1}\left(\begin{array}{c}
\gamma, \gamma-1 / 2 \\
1 / 2
\end{array} ; t\right)
$$

являющееся той же функцией, что и (4.10). Отображение $f(w)$ задается явной формулой (4.11). Прямая подстановка показывает, что функция $g(w)=f(w) / \sqrt{w^{2}-1}$ удовлетворяет интегральному уравнению (4.14) как для положительных, так и для отрицательных $\gamma,|\gamma|<1 / 2$.

Двухлепестковое решение. Далее мы рассмотрим двухлепестковую конфигурацию, растущую в верхней полуплоскости и симметричную относительно мнимой оси (рис. 3б).

Положим угол между двумя лепестками равным $2 \beta$. Очевидно, что этот угол удовлетворяет условию $0<\beta<\pi / 2-\alpha$. Теперь функция $V(w)$ имеет четыре двойных полюса. Два из них расположены в точках $w= \pm 1$. Симметрия требует, чтобы два других полюса находились в точках $w= \pm i$. В данном случае функция $V(w)$ вновь однозначно определяется тремя условиями, приведенными в п. 4.1:

$$
V(w)=\frac{16 \alpha}{\pi}\left(1-\frac{\alpha}{\pi}\right) \frac{w^{2}}{\left(w^{2}-1\right)^{2}}-\frac{8 \beta}{\pi}\left(1-\frac{2 \beta}{\pi}\right) \frac{w^{2}}{\left(w^{2}+1\right)^{2}} .
$$

Заметим, что эта функция также соответствует углу $\pi / 2-\beta$. Та же подстановка $w=e^{x}, f(w)=\operatorname{sh}^{1 / 2} x \cdot \psi(x)$, что и выше, приводит уравнение (4.3) к виду

$$
\psi^{\prime \prime}+\frac{\gamma(1-\gamma)}{\operatorname{sh}^{2} x} \psi-\frac{\delta(1-\delta)}{\operatorname{ch}^{2} x} \psi=\frac{1}{4} \psi,
$$

где $\gamma$ - та же, что и в уравнении (4.9), а $\delta=2 \beta / \pi$. Дальнейшей заменой переменных

$$
\psi=2 \operatorname{sh}^{\gamma} x \cdot \operatorname{ch}^{1 / 2-\gamma} x \cdot F\left(\frac{1}{\operatorname{ch}^{2} x}\right), \quad t=\frac{1}{\operatorname{ch}^{2} x},
$$

уравнение приводится к стандартному гипергеометрическому виду

$$
t(1-t) F_{t t}+\left(\frac{1}{2}-(1+\gamma) t\right) F_{t}-\frac{1}{16}((2 \gamma+2 \delta-1)(2 \gamma-2 \delta+1)) F=0
$$

Оно имеет два линейно независимых решения:

$$
\begin{aligned}
& F^{(1)}(t)={ }_{2} F_{1}\left(\begin{array}{c}
(2 \gamma+2 \delta-1) / 4,(2 \gamma-2 \delta+1) / 4 \\
1 / 2
\end{array} t\right) \\
& F^{(2)}(t)=t_{2}^{1 / 2} F_{1}\left(\begin{array}{c}
(2 \gamma+2 \delta+1) / 4,(2 \gamma-2 \delta+3) / 4 \\
3 / 2
\end{array} t\right) .
\end{aligned}
$$

Для функции $\psi$ первое решение дает

$$
\psi(x)=\operatorname{sh}^{\gamma} x \cdot \operatorname{ch}^{1 / 2-\gamma} x \cdot{ }_{2} F_{1}\left(\begin{array}{c}
(2 \gamma+2 \delta-1) / 4,(2 \gamma-2 \delta+1) / 4 \\
1 / 2
\end{array} \operatorname{ch}^{-2} x\right) .
$$


Переходя к функции $f(w)$ и к исходным углам $\alpha, \beta$, имеем

$$
\begin{aligned}
f(w)= & w\left(1-1 / w^{2}\right)^{2 \alpha / \pi}\left(1+1 / w^{2}\right)^{1-2 \alpha / \pi} \times \\
& \times{ }_{2} F_{1}\left(\begin{array}{c}
(\alpha+\beta) / \pi-1 / 2,(\alpha-\beta) / \pi \\
1 / 2
\end{array} \frac{4}{(w+1 / w)^{2}}\right) .
\end{aligned}
$$

Симметрия требует, чтобы конформное отображение было нечетной функцией переменной $w$. Функция, задаваемая равенством (4.19), действительно нечетная, в то время как второе решение $F^{(2)}$ приводит к четной функции. Используя принцип аргумента, мы видим, что при $0<\beta<\alpha<\pi / 2$ у производной $f^{\prime}(w)$ нет нулей во внешности единичного круга. Таким образом, функция $f(w)$, задаваемая формулой (4.19), является конформным отображением для двухлепесткового решения.

Отметим, что полученная функция выглядит несколько проще, если записать ее как функцию переменной $p=w+1 / w$ в верхней полуплоскости:

$$
z(p)=p\left(1-4 p^{-2}\right)^{\alpha / \pi}{ }_{2} F_{1}\left(\begin{array}{c}
(\alpha+\beta) / \pi-1 / 2,(\alpha-\beta) / \pi \\
1 / 2
\end{array} \frac{4}{p^{2}}\right) .
$$

Аналитическое продолжение этой функции в область $|p|<2$ дает

$$
\begin{aligned}
& z(p)=2\left(1-\frac{p^{2}}{4}\right)^{\alpha / \pi}\left[i e^{-i \beta} \frac{\Gamma\left(\frac{1}{2}\right) \Gamma\left(\frac{1}{2}-\frac{2 \beta}{\pi}\right)}{\Gamma\left(\frac{\alpha-\beta}{\pi}\right) \Gamma\left(1-\frac{\alpha+\beta}{\pi}\right)}\left(\frac{p}{2}\right)^{2 \beta / \pi} \times\right. \\
& \times{ }_{2} F_{1}\left(\begin{array}{c}
(\alpha+\beta) / \pi-1 / 2,(\alpha+\beta) / \pi \\
2 \beta / \pi+1 / 2
\end{array} ; \frac{p^{2}}{4}\right)+ \\
& +e^{i \beta} \frac{\Gamma\left(\frac{1}{2}\right) \Gamma\left(\frac{2 \beta}{\pi}-\frac{1}{2}\right)}{\Gamma\left(\frac{\alpha+\beta}{\pi}-\frac{1}{2}\right) \Gamma\left(\frac{1}{2}-\frac{\alpha-\beta}{\pi}\right)}\left(\frac{p}{2}\right)^{1-2 \beta / \pi} \times \\
& \left.\times{ }_{2} F_{1}\left(\begin{array}{c}
(\alpha-\beta) / \pi+1 / 2,(\alpha-\beta) / \pi \\
3 / 2-2 \beta / \pi
\end{array} \frac{p^{2}}{4}\right)\right] .
\end{aligned}
$$

Граница двухлепестковой структуры в верхней полуплоскости дается функцией $z(p+i 0)$ для $-2<p<2$.

В случае $\beta=\alpha$ гипергеометрическая функция в (4.20) становится тривиальной (равна 1). Соответствующее конформное отображение оказывается отображением на область с разрезами: функция $z(p)=p\left(1-4 p^{2}\right)^{\alpha / \pi}$ отображает верхнюю полуплоскость на верхнюю полуплоскость, разрезанную вдоль двух прямых отрезков, начинающихся в нуле и идущих в северо-восточный и северо-западный квадранты под углом $\alpha$ к действительной оси. В действительности это означает, что $\beta$ равно $\pi / 2-\alpha$, а не $\alpha$, но, как уже отмечалось, функция (4.16) в обоих случаях совпадает.

Некоторые типичные двухлепестковые решения показаны на рис. 5. Нужно различать следующие случаи.

1. Пусть $\alpha \in(0, \pi / 4)$. Тогда:

при $\beta \in(0, \alpha)$ лепестки имеют вид, изображенный на рис. $5 \mathrm{a}$, при $\beta \rightarrow \alpha-0$ они становятся очень тонкими;

при $\beta=\alpha$ лепестки вырождаются в отрезки (рис. 5б);

при $\beta \in(\alpha, \pi / 2-\alpha)$ решение не существует вследствие того, что отображение не является конформным. 

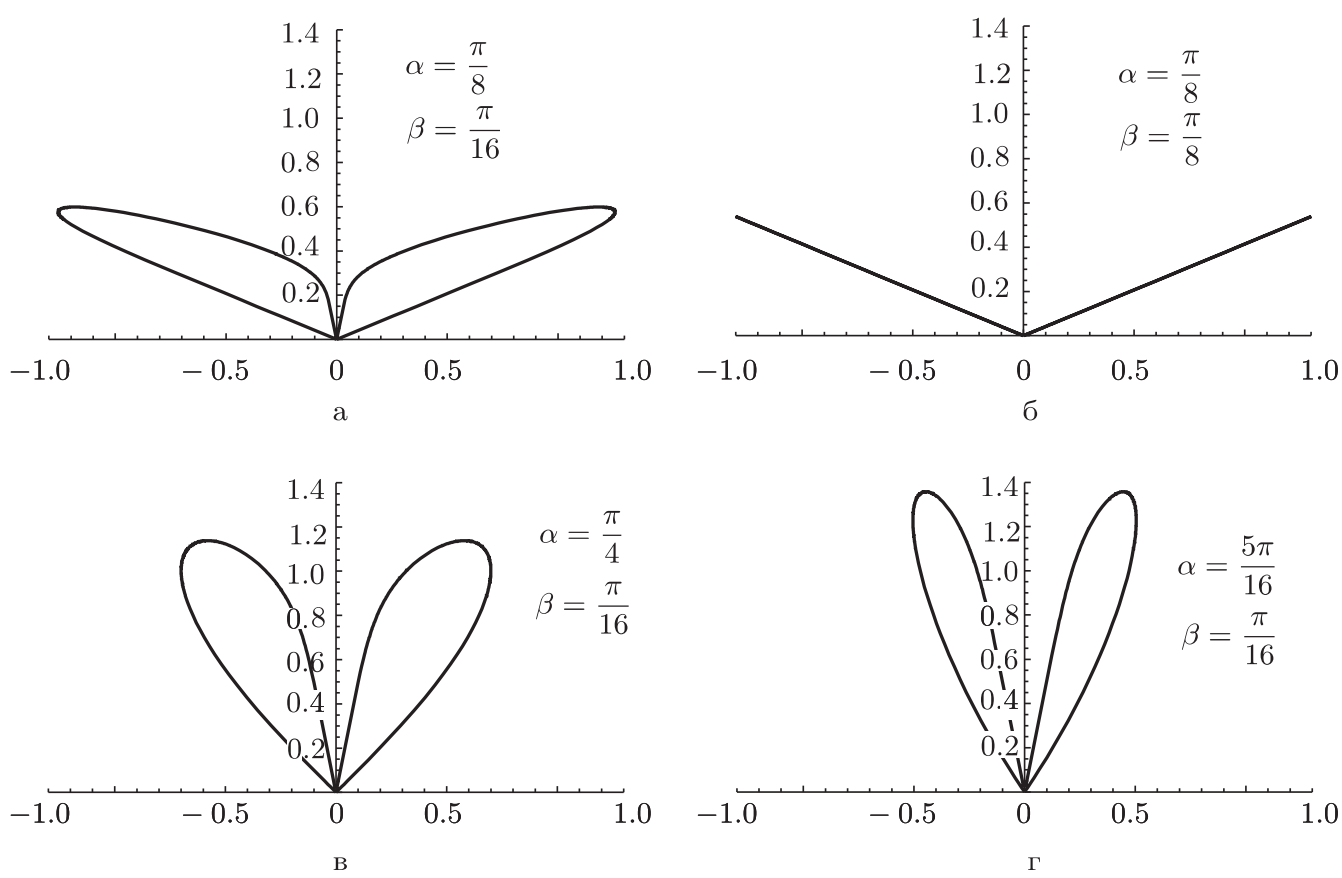

Рис. 5. Двухлепестковые решения при различных $\alpha$ и $\beta$.

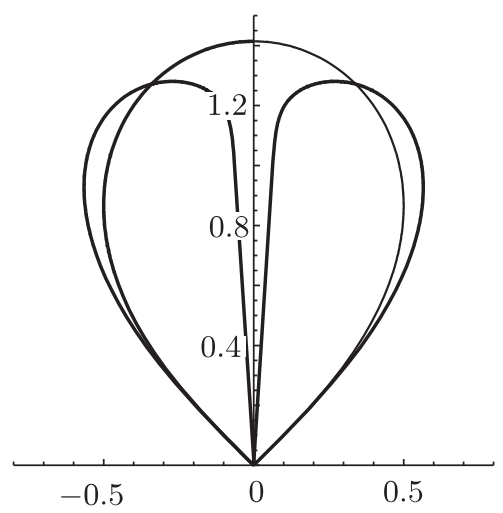

Рис. 6. Однолепестковое решение при $\alpha=\pi / 4$ и двухлепестковое решение при $\alpha=\pi / 4$ и $\beta=\pi / 50$.

2. Пусть $\alpha=\pi / 4, \beta \in(0, \pi / 4)$. Тогда функция $f(w)$ выражается через элементарные функции:

$$
f(w)=(w+1 / w)\left(1-\frac{4}{(w+1 / w)^{2}}\right)^{1 / 4} \cos \left[2\left(\frac{1}{4}-\frac{\beta}{\pi}\right) \arcsin \left(\frac{2}{w+1 / w}\right)\right]
$$

типичный вид этой функции представлен на рис. 5в, решение вырождается при $\beta=\pi / 4$. 
3. Пусть $\alpha \in(\pi / 4, \pi / 2)$. Тогда:

при $\beta \in(\alpha, \pi / 2)$ лепестки имеют вид, изображенный на рис. 5г;

при $\beta=\pi / 2-\alpha$ лепестки вырождаются в отрезки;

при $\beta \in(\pi / 2-\alpha, \alpha)$ решение не существует, так как отображение не является конформным.

Также отметим, что форма двухлепестковых решений при малых $\beta$ становится близкой к форме однолепестковых решений с тем же $\alpha$ (рис. 6), что и следовало ожидать исходя из вида дифференциального уравнения.

Благодарности. Авторы хотели бы выразить благодарность П.Б. Вигману, М. Б. Минееву-Вайнштейну и Д. Хавинсону за ценные обсуждения. Эта работа была выполнена при частичной поддержке РФФИ (грант № 08-02-00287), Программы поддержки ведущих научных школ (грант НШ-3035.2008.2) и Федерального агентства по науке и инновациям (госконтракт № 02.740.11.5029). Также работа была частично поддержана РФФИ (совместные гранты № 09-02-90493-Ukr, 09-02-93105-CNRSL (Д. В. Васильев) и 09-01-92437-СЕ_а (А. В. Забродин)).

\section{Список литературы}

[1] A. Zabrodin, J. Phys. A, 42:8 (2009), 085206, 23 pp., arXiv: 0811.4054.

[2] S. D. Howison, European J. Appl. Math., 3:3 (1992), 209-224.

[3] P. J. Davis, The Schwarz function and its applications, The Carus Math. Monogr., 17, The Math. Association of America, Buffalo, N.Y., 1974.

[4] H. Thomé, M. Rabaud, V. Hakim, Y. Couder, Phys. Fluids A, 1:2 (1989), 224-240.

[5] M. Ben Amar, Phys. Rev. A, 43:10 (1991), 5724-5727; 44:6 (1991), 3673-3685.

[6] Y. Tu, Phys. Rev. A, 44:2 (1991), 1203-1210.

[7] R. Combescot, Phys. Rev. A, 45:2 (1992), 873-884.

[8] I. Markina, A. Vasil'ev, Sci. Ser. A, 9 (2003), 33-43; European J. Appl. Math., 15:6 (2004), 781-789.

[9] Ar. Abanov, M. Mineev-Weinstein, A. Zabrodin, Phys. D, 235:1-2 (2007), 62-71, arXiv: nlin/0606068.

[10] А. И. Маркушевич, Теория аналитических функиий, т. 2: Далънейшее построение теории, Наука, М., 1968. 